\title{
Design and theoretical analysis of a clock jitter reduction circuit using gated phase blending between self-delayed clock edges
}

\author{
Kiichi Niitsu $^{1 \text { a) }}$, Osamu Kobayashi ${ }^{2}$, Takahiro J. Yamaguchi ${ }^{3}$, and Haruo Kobayashi ${ }^{2}$
}

\begin{abstract}
This study demonstrates the design and theoretical analysis of a clock jitter reduction circuit that exploits the phase blending technique between the uncorrelated clock edges that are self-delayed by multiples of the clock cycle, $n T$. By blending uncorrelated clock edges, the output clock edges approach the ideal timing and, thus, timing jitter can be reduced by a factor of $\sqrt{2}$ per stage. There are three technical challenges to realize this: 1) generating uncorrelated clock edges, 2) phase averaging with small time offset from the ideal center position, and 3) minimizing the error in $n T$-delay being deviated from ideal $n T$. The proposed circuit overcomes each of these by exploiting an $n T$-delay, gated phase blending, and self-calibrated $n T$-delay elements, respectively. Measurement results with a 180-nm CMOS prototype chip demonstrated an approximately four-fold reduction in timing jitter from $30.2 \mathrm{ps}$ to $8.8 \mathrm{ps}$ in $500-\mathrm{MHz}$ clock by cascading the proposed circuit with four-stages. Theoretical analysis for evaluating the limit of jitter reduction is also presented. Keywords: jitter, jitter reduction, phase blending, self-delayed, PLL Classification: Integrated circuits
\end{abstract}

\section{Introduction}

Jitter has been becoming one of the most critical issues in state-of-the-art clocking VLSI systems, and this has motivated us to focus on low-jitter PLLs. In typical cases, there are two candidate designs for a PLL: one with an LC-VCO and another with a ring VCO. The latter has the advantage of a lower footprint, but it does not achieve a comparably good jitter performance. Here we propose a clock jitter reduction circuit that overcomes this trade-off: a low-jitter PLL with a small-footprint ring VCOs.

Until now, two approaches to designing jitter reduction circuits have been reported. One is a jitter cleaner circuit using narrow-band cascaded PLLs [1] and another is an anti-jitter circuit exploiting an integer storage capacitor [2]. The former has a larger footprint, but the latter has lower robustness against process variation because it uses a comparator. The proposed circuit outperforms both these conventional designs. Moreover, it requires only CMOS logic gates for implementation, making it be cost effective, and employs self-calibration technique for realizing high robustness against process variations. In addition to these

${ }^{1}$ Graduate School of Engineering, Nagoya University, 4648601, Japan

${ }^{2}$ STARC, Japan

${ }^{3}$ Graduate School of Engineering, Gunma University, Japan

a) niitsu@nuee.nagoya-u.ac.jp

DOI: $10.1587 /$ elex.16.20190218

Received April 3, 2019

Accepted April 17, 2019

Publicized May 17, 2019

Copyedited July 10, 2019 merits, the proposed design is scalable in terms of the number of stages, which is an advantage when employed as a pre-driver for ADCs and DACs. That is, a large number of stages can be implemented, allowing the clock to be fed into high-resolution data converters.

This paper describes the design of the low-cost, highPVT-robust jitter reduction scheme and presents measurement results that support the claims. Although a limited case of this study was reported in a previous work as a conference proceeding [3], this study demonstrates detail of the circuit implementation and presents the theoretical analysis of general cases for the first time. The reminder of this paper is organized as follows. In this paper, the measurement section is spread to some parts. Section II describes the proposed clock jitter reduction circuit including the test chip design and its measured results. Section III presents theoretical analysis for evaluating the limitation of the proposed scheme. Section IV concludes the paper.

\section{Clock jitter reduction circuit using gated phase blending between self-delayed clock edges}

The mechanism and strategy of the proposed clock jitter reduction using gated phase blending between self-delayed clock edges are explained mathematically and experimentally in this section. After presenting the mechanism of clock jitter reduction, three key techniques are introduced.

\subsection{Mechanism of clock jitter reduction}

Fig. 1 illustrates the conceptual image of the proposed clock jitter reduction circuit using a phase averaging between uncorrelated self-delayed clock edges. By averaging the phase of uncorrelated input and $n T$-delayed clock, timing jitter can be reduced in each cycle. Since this technique is based on phase averaging, it is not effective to periodic jitter. For reducing the periodic jitter, another technique is required. As stated in previous reports $[4,5]$, a large delay line weakens correlation between edges in ring oscillators. However, the delay line causes requirement of additional power consumption. Furthermore, this delay line causes additional jitter, which limits the total jitter reduction performance.

Fig. 2 shows a conceptual diagram of the proposed circuit. Each stage of the circuit consists of a self-calibrated $n T$-delay and the newly proposed gated phase blender. The self-calibrated $n T$-delay generates uncorrelated clock edges. The gated phase blender averages these edges to output a low-jitter clock signal. Theoretically, the timing 


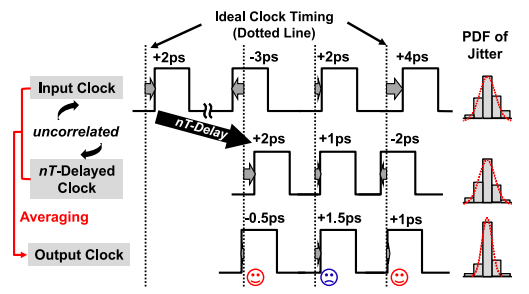

Fig. 1. Conceptual image of the proposed clock jitter reduction circuit using a phase averaging between uncorrelated self-delayed clock edges.

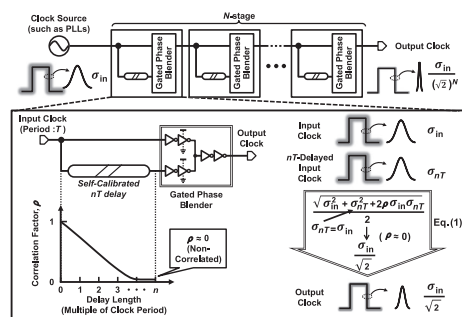

Fig. 2. Concept and theoretical analysis of the proposed clock jitter reduction circuit using a gated phase blending between uncorrelated selfdelayed clock edges. Copyright IEEE 2012 [3, 4].

jitter is reduced by a factor of $\sqrt{2}$. This is given by Eq. (1), shown on the right side in Fig. 2 and below:

$\frac{\sqrt{\sigma_{i n}^{2}+\sigma_{n T^{2}}+2 \rho \sigma_{i n} \sigma_{n T}}}{2} \rightarrow \frac{\sigma_{\text {in }}}{\sqrt{2}}\left(\right.$ where, $\left.\sigma_{n T} \approx \sigma_{i n}, \rho \approx 0\right)$

where a smaller correlation coefficient, $\rho$, is required for realizing a small timing jitter. Note that $\sigma_{\text {in }}$ and $\sigma_{n T}$ are the RMS values of the timing jitter of the input clock and its $n T$-delayed clock, respectively. Moreover, it is assumed that both input signals have approximately the same standard deviation, $\sigma$, and the $n T$-delay is designed to minimize the delay deviation as explained later.

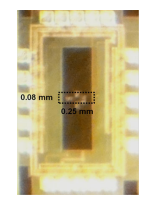

Fig. 3. Test chip microphotograph. Copyright IEEE 2012 [3, 4].

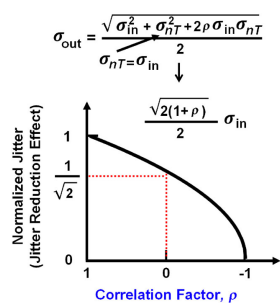

Fig. 4. Theoretical overview of the $n T$-delay for generating uncorrelated clock edges.

In summary, there are the three key techniques for enabling the clock jitter reduction using gated phase blending between self-delayed edges. The first one is $n T$-delay for generating uncorrelated clock edges. If the two clock edges for phase blending are correlated $(\rho=1)$, there is no meaning to do phase averaging and no jitter reduction can be obtained. We have implemented $n T$-delay for generating uncorrelated clock edges.

\subsection{Test chip design}

In order to investigate the effectiveness of the proposed circuit architecture, we have designed the test chip. For designing test chip, some EDAs have been utilized. For circuit entry and mask layout, Cadence design tool chain (Cadence IC 61) has been employed. For circuit simulation, Cadence Spectre has been employed. For physical verification, Mentor Calibre has been employed.

\subsection{Test chip prototyping and measurement setup}

In order to verify the effectiveness of the proposed technique, a test chip was fabricated using 180-nm CMOS technology. The footprint of this four-stage implementation was $0.08 \mathrm{~mm} \times 0.25 \mathrm{~mm}$, including all sub-blocks such as the self-calibration circuit (Fig. 3). The input clock is fed to the circuit by a BERT (Agilent 81250). Timing jitters were measured with an oscilloscope (Tektronix DSA71254B).

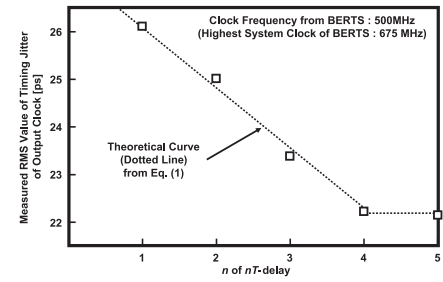

Fig. 5. Measured timing jitter at the minimum point dependence on the number of clock cycles, $n$ of $n T$-delay elements. Theoretical curve is obtained from Eq. (1) and estimation that the required number of stages is four. Copyright IEEE $2012[3,4]$.

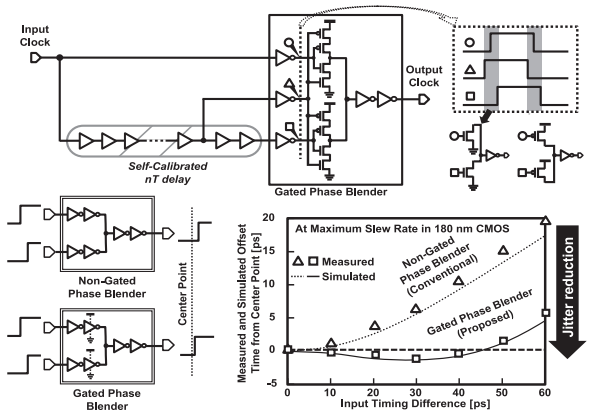

Fig. 6. Proposed gated phase blender for phase averaging with small time offset. Copyright IEEE 2012 [3, 4].

\section{4 $\boldsymbol{n} \boldsymbol{T}$-delay for generating uncorrelated clock edges}

Fig. 4 shows the theoretical overview of $n T$-delay for generating uncorrelated clock edges. As described in the figure, when there is no correlation, the jitter reduction effect is $1 / \sqrt{2}$. While, if the correlation factor is -1 , jitter can be perfectly deleted.

Fig. 5 shows the measured timing jitter at the minimum points dependence on the number of clock cycles, $n$ of $n T$-delay elements. Longer $n T$-delay weakens the correlation coefficient, and hence, the timing jitter, as predicted by Eq. (1). With the measurement setup used in this study, the correlation coefficient decreased when the num- 
ber of multiples of $n T$-delay, $n$, increased up to four and it saturated when $n$ was greater than four. Therefore, the $n T$ delay was selected as four: $n=4$.

\subsection{Gated phase blender for achieving small time offset} Fig. 6 shows the proposed gated phase blender for achieving a small offset time from the center position. The phase blender is necessary for achieving phase averaging. In order to achieve phase averaging ideally, the designer must minimize the offset from the center point. Due to the offset, the output jitter increases significantly. The triangle and square points are measured results and dotted. While, the solid lines are simulated results.

By gating the PMOS or NMOS before phase blending, penetration currents can be eliminated and thus the offset time from the ideal center position can be minimized. Unlike the previous approach [6], the two input paths must have the identical electrical length since it is unknown which pulse comes first. The control signal for gating is externally supplied by tapping the self-calibrated $n T$-delay. The upper right part of the figure shows the conceptual operation image. This image is depicted on not the absolute timing but the phase. Thus, the triangle signal comes between the circle and square signal. Since the triangle signal cannot come previous timing than the others at the initial operation, gated operation can function after several clock edges.

The offset reduction was confirmed in both SPICE simulations and measurements with a test chip. In order to minimize the additional jitter of the phase blending circuit, which is caused by the power supply noise, the slew rate was designed to be as high as possible.

In order to implement the proposed gated phase blender, the additional footprint and power is required. But, they are negligible and do not dominate the overall clock jitter reduction circuit. Since the proposed circuit consists of standard inveter cell (one PMOS and one NMOS), it may not degrade the operational frequency in the usual conditions. Therefore, the gated phase blender has advantage from the viewpoint of additional cost and jitter reduction effect.

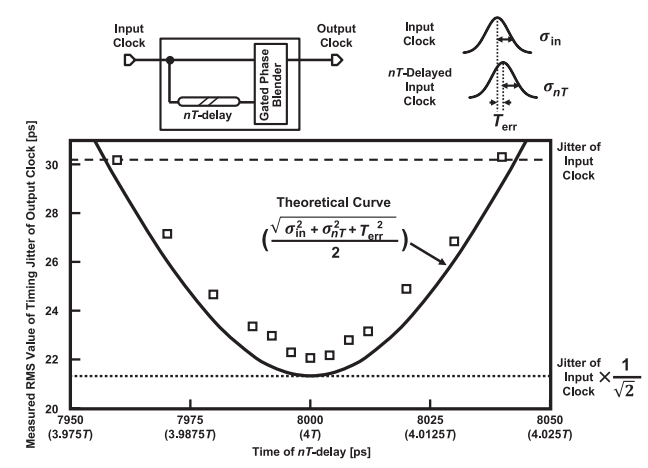

Fig. 7. Measured RMS value of timing jitter as a function of the duration of the $n T$-delay and comparison with its theoretical curve. Copyright IEEE $2012[3,4]$.

\subsection{Self-calibrated $n T$-delay for minimizing the error in $n T$-delay}

Fig. 7 shows the measured RMS value of the timing jitter as a function of the error in $n T$-delay. The difference between Fig. 5 and Fig. 7 is as follows: the results in Fig. 5 shows the jitter at the minimum point (at the minima without any timing error), while the results in Fig. 7 shows not only at the minimum point but also the surrounded points. It can be seen that a smaller error in $n T$-delay leads to more efficient jitter reduction. The measured results match well with the theoretical curves obtained by taking the error in $n T$-delay into consideration.

In order to reduce jitter, the error in $n T$-delay, $T_{\text {err }}$ must be kept smaller than $\sqrt{2}$-times $\sigma_{\text {in }}$ :

$\frac{\sqrt{\sigma_{i n}^{2}+\sigma_{n T^{2}+T_{e r r}^{2}}}}{2}<\sigma_{\text {in }} \Leftrightarrow T_{\text {err }}<\sqrt{2} \sigma_{\text {in }}$ (where, $\left.\sigma_{n T} \approx \sigma_{\text {in }}\right)$

Furthermore, the reported self-calibrated delay elements [7] enable the proposed circuit to further reduce input jitter. However, due to imperfections in the phase blender and jitter accumulation in $n T$-delay, the measured jitter was larger than the theoretical value. This limitation is discussed in the next section.

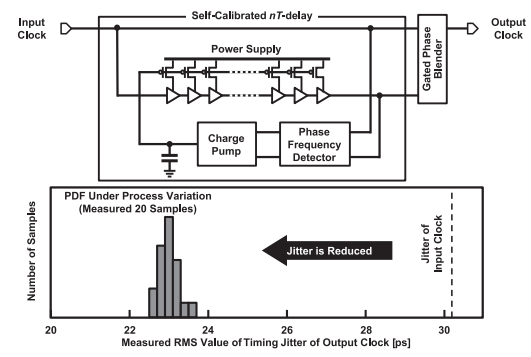

Fig. 8. Schematic of the self-calibrated $n T$-delay and measured PDF of the number of samples as a function of measured RMS value of timing jitter of output clock. Copyright IEEE 2012 [3, 4].

A schematic and measurement results for the selfcalibrated $n T$-delay are shown in Fig. 8. It verifies that the circuit can provide high robustness against process variations. The self-calibrated $n T$-delay consists of a phase frequency detector and a charge pump. Despite a simple circuit implementation, the self-calibrated scheme was confirmed to be effective in minimizing the error in $n T$ delay, and thus allows both jitter reduction and high robustness against process.

\subsection{Multi-stage configuration for scalability and further jitter reduction}

By configuring the proposed clock jitter reduction (CJR) circuits in multi-stage, scalability and further jitter reduction can be obtained as shown in Fig. 10. The proposed design is scalable in terms of the number of stages, which is an attractive advantage when employed as a pre-driver for ADCs and DACs. That is, a large number of stages can be implemented, allowing the clock to be fed into highresolution data converters as illustrated in Fig. 9.

The measured results, including timing jitter probability distribution functions (PDFs), with a four-stage configuration are presented in Fig. 10. As the input signal, the net 


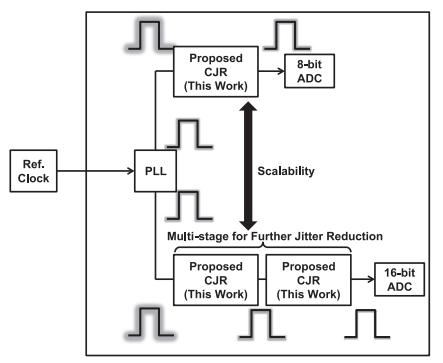

Fig. 9. Conceptual diagram of the scalability of the proposed circuit

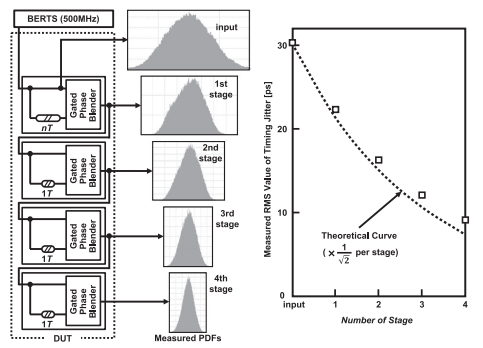

Fig. 10. Measurement setup and measured PDFs, together with experimental plot showing the dependence of the RMS value of the timing jitter on the number of stages. Copyright IEEE 2012 [3, 4].

before the gated phase blender was tapped and measured. After propagating through the first stage, adjacent positive clock edges are uncorrelated with each other. Consequently the length of the $n T$-delay in the second and later stages can be minimized. Moreover, smaller $n T$-delay elements contribute to both footprint reduction and smaller jitter accumulation over the $n T$-delay. Therefore, high jitter reduction can be realized. The results, which are shown in Fig. 10, validates that the timing jitter of the injected $500-\mathrm{MHz}$ clock was successfully reduced by a factor of three. Note that the $500-\mathrm{MHz}$ clock was selected in order not to exceed the maximum system clock frequency of BERT $(675 \mathrm{MHz})$. However, this frequency is far below the potential operating frequency of the proposed circuit. Instead a CML-based implementation without PMOS was implemented, which operates at $3.35 \mathrm{GHz}$, the maximum frequency of the BERT used in this study. Since PMOS can be eliminated, there is no penetration current and gated operation becomes unnecessary.

\section{Theoretical analysis for evaluating the limitation}

\subsection{Theoretical background}

In order to evaluate the limitation of the proposed jitter reduction, theoretical analysis was performed. The jitter reduction performance is limited by mainly two reasons. One is jitter in $n T$-delay, and the other is imperfection of period of $n T$-delay. In this section, the theoretical investigation of the limitation due to the jitter in the $n T$-delay is provided.

\subsection{Limitation due to jitter of $\boldsymbol{n T}$-delay}

In (1), we have assumed that the jitter of before and after propagation in $n T$-delay is equivalent. However, when taking the additional jitter of the $n T$-delay into account, this assumption cannot be applied and (1) can be expressed as follows:

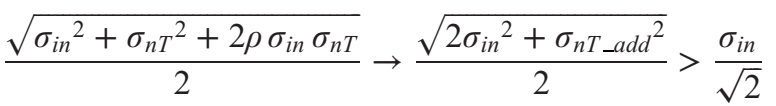

$$
\begin{aligned}
& \text { (where, } \sigma_{n T}=\sigma_{\text {in }}, \rho \approx 0 \text { ) }
\end{aligned}
$$

Note that $\sigma_{\text {nT_add }}$ is the additional jitter of the $n T$-delay and defined as followings:

$$
\sigma_{n T \_a d d}^{2}=\sigma_{n T}{ }^{2}-\sigma_{i n}^{2}
$$

Equation (3) implies that the output jitter may exceed the input jitter in some condition. Thus, jitter reduction cannot be achieved due to the additional jitter of the $n T$-delay when the following condition:

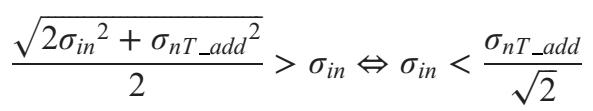

This analysis implies that the designers have to consider the above theoretical limitation when using the proposed method.

\subsection{Increase of power consumption}

The proposed circuit can be used associated with the original PLL at the expense of additional power consumption. In this section, we discuss about the impact of this additional power consumption on the total system performance.

The simulated total power consumption of the proposed circuit under $500 \mathrm{MHz}$ operation is $2.12 \mathrm{~mW}$. The power consumption of the delay line $(n=4)$, the gated phase blender, and the calibration circuit in the self-calibrated delay line are $1.44 \mathrm{~mW}, 0.06 \mathrm{~mW}$, and $0.62 \mathrm{~mW}$, respectively.

In order to consider the advantage of the proposed architecture, we have estimated the use case with the ringVCO based PLL in the same technology node $(180 \mathrm{~nm}$ CMOS) [9]. The PLL in [9] consumes power of $6.1 \mathrm{~mW}$ totally. The analog part consumes $3.83 \mathrm{~mW}$ and the digital part consumes $2.27 \mathrm{~mW}$, respectively.

The following FoM of the PLL [10] was utilized for the estimation.

$$
\text { FoM }=10 \log \left[\left(\frac{\sigma_{R M S}}{1 \mathrm{~s}}\right)^{2}\left(\frac{\text { Power }}{1 \mathrm{~mW}}\right)\right]
$$

From this equation, in order to reduce jitter value while keeping same FoM, $\sigma_{R M S}{ }^{2} \times$ Power must be constant.

By considering 1-stage application of the proposed technique to the PLL in [9], we can estimate the advantage as follows. Since the jitter reduction effect of the proposed circuit is $1 / \sqrt{2}$, power overhead of $6.1 \mathrm{~mW}$ is acceptable. While, the overhead of the proposed technique is only $2.12 \mathrm{~mW}$, which is much less than the acceptable power overhead. Performance comparison including recent progress [11] has been summarized in Table I.

According to the above estimation, the proposed technique has advantage when applying to the PLL in [9]. Since the proposed circuit is based on the digital circuitry, it is familiar with the technology scaling. Thus, even in the more scaled CMOS technology, the proposed technique will be effective. 
Table I. Performance comparison.

\begin{tabular}{|c|c|c|c|}
\hline & JSSC'09 [10] & CICC'18 [11] & This work \\
\hline Frequency & $2.2 \mathrm{GHz}$ & $2.4 \mathrm{GHz}$ & N/A \\
\hline $\begin{array}{c}\text { Original } \\
\text { power }\end{array}$ & $6.1 \mathrm{~mW}$ & $6.1 \mathrm{~mW}$ & N/A \\
\hline $\begin{array}{c}\text { Required } \\
\text { total power } \\
\text { for same } \\
\text { FoM }\end{array}$ & $12.2 \mathrm{~mW}$ & $12.2 \mathrm{~mW}$ & N/A \\
\hline $\begin{array}{c}\text { Required } \\
\text { additional } \\
\text { power for } \\
\text { same FoM }\end{array}$ & $6.1 \mathrm{~mW}$ & $6.1 \mathrm{~mW}$ & $2.12 \mathrm{~mW}$ \\
\hline
\end{tabular}

\subsection{Increase of area}

Since the proposed clock jitter reduction circuit employs a charge pump and the capacitor, additional area is required and must be discussed. In the implementation of this study, we minimize the sizes of charge pump and the capacitor as small as possible. Thus, required footprint can be reduced to $0.02 \mathrm{~mm}^{2}$.

\subsection{Phase noise and spectrum measurement}

Due to the limitation of measurement setup, we did not get phase noise and spectrum information. However, measurement and analysis of the phase noise and spectrum at the output of each stage is beneficial for better understanding.

\subsection{Other techniques for jitter reduction}

There are some other techniques for jitter reduction. For instance, delay-discriminator-based active phase-noise cancellation scheme has been presented in previous work [12]. Compared to the conventional work, the proposed technique has advantage from the viewpoint of small additional footprint while disadvantage of reduced jitter reduction effect. These techniques will be also helpful for developing energy-efficient biomedical IoTs [13-32].

\section{Conclusion}

In this paper, we reported on design and theoretical analysis of a clock jitter reduction circuit using gated phase blending between self-delayed clock edges. Measurement results with a prototype chip in 180-nm CMOS successfully demonstrated an approximate four-fold reduction in timing jitter from $30.2 \mathrm{ps}$ to $8.8 \mathrm{ps}$ in $500-\mathrm{MHz}$ clock by cascading the proposed circuit with four-stages. The limitation of the proposed scheme was theoretically investigated for practical application. Theoretical investigation of limitation was also discussed.

\section{Acknowledgments}

This research was financially supported by PRESTO, JST, by a Grant-in-Aid for Scientific Research (S) (Nos. 20226009, 25220906), Grants-in-Aid for Young Scientists (A) (No. 16H06088) from the Ministry of Education, Culture, Sports, Science and Technology of
Japan, by the Strategic Information and Communications R\&D Promotion Programme (Nos. 121806006, 152106004) of the Ministry of Internal Affairs and Communications, Japan, by TOYOTA RIKEN, and by The Nitto Foundation. The fabrication of CMOS chips was supported by Taiwan Semiconductor Manufacturing Co., Ltd. (TSMC, Taiwan), and the VLSI Design and Education Center (VDEC), University of Tokyo in collaboration with Synopsys, Inc. and Cadence Design Systems, Inc.

\section{References}

[1] Texas Instrument's Production Introduction Page: http://www. ti.com/lsds/ti/clocks-timers/clock-jitter-cleaners-products.page.

[2] M. J. Underhill: US patent 6,791,393 B1 (Sep. 14, 2004).

[3] K. Niitsu, et al.: "A clock jitter reduction circuit using gated phase blending between self-delayed clock edges," Proc. IEEE Symp. on VLSI Circuits (2012) 142 (DOI: 10.1109/VLSIC.2012.6243830).

[4] K. Niitsu, et al:: "Design of a clock jitter reduction circuit using gated phase blending between self-delayed clock edges," Proc. IEEE/ACM Asia and South Pacific Design Automation Conf. (2013) 103 (DOI: 10.1109/ASPDAC.2013.6509577).

[5] J. A. McNeill: "Jitter in ring oscillators," IEEE J. Solid-State Circuits 32 (1997) 870 (DOI: 10.1109/4.585289).

[6] K. Niitsu, et al.: "CMOS circuits to measure timing jitter using a self-referenced clock and a cascaded time difference amplifier with duty-cycle compensation,” IEEE J. Solid-State Circuits 47 (2012) 2701 (DOI: 10.1109/JSSC.2012.2211655).

[7] B. W. Garlepp, et al.: "A portable digital DLL for high-speed CMOS interface circuits," IEEE J. Solid-State Circuits 34 (1999) 632 (DOI: 10.1109/4.760373).

[8] W. Khalil, et al.: "A self-calibrated on-chip phase-noise measurement circuit with $-75 \mathrm{dBc}$ single-tone sensitivity at $100 \mathrm{kHz}$ offset," IEEE J. Solid-State Circuits 42 (2007) 2758 (DOI: 10.1109/JSSC.2007.908689).

[9] X. Yu, et al:: "An FIR-embedded noise filtering method for delta sigma fractional-N PLL clock generators," IEEE J. Solid-State Circuits 44 (2009) 2426 (DOI: 10.1109/JSSC.2009.2021086).

[10] J. Zhu, et al:: "A $0.0021 \mathrm{~mm}^{2} 1.82 \mathrm{~mW} 2.2 \mathrm{GHz}$ PLL using timebased integral control in $65 \mathrm{~nm}$ CMOS," IEEE J. Solid-State Circuits 52 (2017) 8 (DOI: 10.1109/JSSC.2016.2598768).

[11] S. S. Nagam and P. R. Kinget: “A $0.008 \mathrm{~mm}^{2} 2.4 \mathrm{GHz}$ type-I subsampling ring-oscillator-based phase-locked loop with a $-239.7 \mathrm{~dB}$ FoM and $-64 \mathrm{dBc}$ reference spurs," Proc. IEEE Custom Integrated Circuits Conference (2018) 1 (DOI: 10.1109/CICC.2018.8357091).

[12] S. Min, et al.: "A 90-nm CMOS 5-GHz ring-oscillator PLL with delay-discriminator-based active phase-noise cancellation," IEEE J. Solid-State Circuits 48 (2013) 1151 (DOI: 10.1109/JSSC.2013. 2252515).

[13] K. Niitsu: "Energy-autonomous biosensing platform using supplysensing CMOS integrated sensor and biofuel cell for nextgeneration healthcare Internet of Things," Jpn. J. Appl. Phys. 57 (2018) 1002A5 (DOI: 10.7567/JJAP.57.1002A5).

[14] K. Hayashi, et al.: “A $385 \times 385 \mu \mathrm{m} 20.165 \mathrm{~V} 0.27 \mathrm{nW}$ fullyintegrated supply-modulated OOK transmitter in $65 \mathrm{~nm}$ CMOS for glasses-free, self-powered, and fuel-cell-embedded continuous glucose monitoring contact lens," accepted to IEICE Trans. Electron. (2019).

[15] K. Hayashi, et al.: "An FSK inductive-coupling transceiver using $60 \mathrm{mV} 0.64 \mathrm{fJ} /$ bit $0.0016 \mathrm{~mm}^{2}$ load-modulated transmitter and LCoscillator-based receiver in $65 \mathrm{~nm}$ CMOS for energy-budgetunbalanced application," accepted to IEICE Trans. Electron. (2019).

[16] S. Arata, et al:: "Yield and open-circuit-voltage enhancement of $0.36 \mathrm{~mm}^{2}$ solid-state CMOS-compatible glucose fuel cell by using repeated separator coating," accepted to Jpn. J. Appl. Phys. (DOI: 10.7567/1347-4065/aafc9e).

[17] Y. Nishio, et al.: "Design and calibration of a small-footprint, lowfrequency, and low-power gate leakage timer using differential leakage technique," IEICE Trans. Electron. (2019) (DOI: 10.1587/ 
transele.2018CDP0005).

[18] K. Niitsu, et al.: "A 65-nm CMOS fully-integrated analysis platform using an on-chip vector network analyzer and a transmission-line-based detection window for analyzing circulating tumor cell and exosome," accepted to IEEE Trans. Biomed. Circuits Syst. (DOI: 10.1109/TBCAS.2018.2882472).

[19] M. Matsunaga, et al:: "Design and analysis of a three-dimensional millimeter-wave frequency-shift based CMOS biosensor using vertically stacked spiral inductors in LC oscillators," accepted to Analog Integr. Circ. Sig. Process. 98 (2019) 453 (DOI: 10.1007/ s10470-018-1267-5).

[20] K. Hayashi, et al.: “A $6.1 \mathrm{nA}$ fully-integrated CMOS supplymodulated OOK transmitter in $55 \mathrm{~nm}$ DDC CMOS for glass-free, self-powered, and fuel-cell-embedded continuous glucose monitoring contact lens," accepted to IEEE Trans. Circuits Syst. II, Exp. Briefs 65 (2018) 1360 (DOI: 10.1109/TCSII.2018.2860636).

[21] K. Niitsu, et al:: "A self-powered supply-sensing biosensor platform using bio fuel cell and low-voltage, low-cost CMOS supply-controlled ring oscillator with inductive-coupling transmitter for healthcare IoT," IEEE Trans. Circuits Syst. I, Reg. Papers 65 (2018) 2784 (DOI: 10.1109/TCSI.2018.2791516).

[22] S. Arata, et al.: "Wafer-scale development and experimental verification of $0.36-\mathrm{mm}^{2} 228-\mathrm{mV}$ open-circuit-voltage solid-state CMOS-compatible glucose fuel cell for healthcare IoT application,” Jpn. J. Appl. Phys. 57 (2018) 04FM04 (DOI: 10.7567/ JJAP.57.04FM04).

[23] M. Matsunaga, et al.: "Design trade-off between spatial resolution and power consumption in CMOS biosensor circuit based on millimeter-wave LC-oscillator array,” Jpn. J. Appl. Phys. 57 (2018) 03EC02 (DOI: 10.7567/JJAP.57.03EC02).

[24] T. Nakanishi, et al: "A 40-GHz fully integrated circulating tumor cell analysis vector network analyzer in 65-nm CMOS technology with coplanar-line-based detection area," Jpn. J. Appl. Phys. 57 (2018) 03EC01 (DOI: 10.7567/JJAP.57.03EC01).

[25] K. Itakura, et al.: "Theoretical analysis and simulation study of low-power CMOS electrochemical impedance spectroscopy biosensor in $55 \mathrm{~nm}$ DDC technology for cell-state monitoring," Jpn. J. Appl. Phys. 57 (2018) 01AG02 (DOI: 10.7567/JJAP.57.01AG02).

[26] A. Kobayashi, et al.: "Design and experimental verification of $0.19 \mathrm{~V} 53 \mu \mathrm{W} 65 \mathrm{~nm}$ CMOS integrated supply-sensing sensor with a supply-insensitive temperature sensor and inductive-coupling transmitter for a self-powered bio-sensing using a biofuel cell," IEEE Trans. Biomed. Circuits Syst. 11 (2017) 1313 (DOI: 10.1109/ TBCAS.2017.2735447).

[27] K. Ikeda, et al.: "Design and electrochemical measurement of a current-mode analog-to-time converter with short-pulse output capability using local intra-cell activation for high-speed timedomain biosensor array," Analog Integr. Circ. Sig. Process. 92 (2017) 403 (DOI: 10.1007/s10470-017-1003-6).

[28] K. Gamo, et al:: "A current-integration-based CMOS amperometric sensor with $1024 \times 1024$ bacteria-sized microelectrode array for high-sensitivity bacteria counting," IEICE Trans. Electron. E100-C (2017) 602 (DOI: 10.1587/transele.E100.C.602).

[29] K. Ikeda, et al.: "Design and analysis of scalability in current-mode analog-to-time converter for an energy-efficient and high-resolution CMOS biosensor array," IEICE Trans. Electron. E100-C (2017) 597 (DOI: 10.1587/transele.E100.C.597).

[30] Y. Yamaji, et al.: "Sub-1-V CMOS-based electrophoresis using electroless gold plating for small-form-factor biomolecule manipulation," IEICE Trans. Electron. E100-C (2017) 592 (DOI: 10. 1587/transele.E100.C.592).

[31] K. Niitsu, et al:: "Development of microelectrode arrays using electroless plating for CMOS-based direct counting of bacterial and HeLa cells," IEEE Trans. Biomed. Circuits Syst. 9 (2015) 607 (DOI: 10.1109/TBCAS.2015.2479656).

[32] H. Ishihara, et al:: "Analysis and experimental verification of DNA single base polymerization detection using CMOS FET-based redox potential sensor array," Jpn. J. Appl. Phys. 54 (2015) 04DL05 (DOI: 10.7567/JJAP.54.04DL05). 